\title{
Tubular Biomarkers and Chronic Kidney Disease Progression in SPRINT Participants
}

\author{
Vasantha Jotwani ${ }^{a, b}$ Pranav S. Garimellac, d Ronit Katz ${ }^{\mathrm{e}}$ Rakesh Malhotrac, d \\ Jeffrey Bates ${ }^{f} \quad$ Alfred K. Cheung $^{g}$ Michel Chonchol ${ }^{\text {h }}$ Paul E. Drawz ${ }^{\mathrm{i}}$ \\ Barry I. Freedman' William E. Haleyk Anthony A. Killeen' Henry Punzim \\ Mark J.Sarnak ${ }^{n}$ Mark S. Segal ${ }^{\circ}$ Michael G. Shlipak ${ }^{a, b}$ Joachim H. Ix ${ }^{c, d}$ \\ for the SPRINT Research Group
}

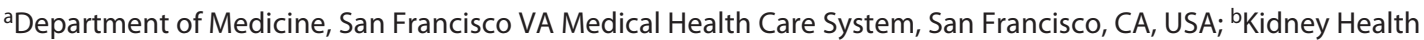
Research Collaborative, San Francisco VA Medical Center and University of California, San Francisco, CA, USA; 'Department of Medicine, University of California, San Diego, CA, USA; dVeterans Affairs San Diego Healthcare System, San Diego, CA, USA; ${ }^{e}$ Kidney Research Institute, University of Washington, Seattle, WA, USA; ${ }^{\prime}$ Michael E. DeBakey VA Medical Center and Baylor College of Medicine, Houston, TX, USA; 9 Department of Medicine, University of Utah, Salt Lake City, UT, USA; h' Department of Medicine, University of Colorado Anschutz Medical Campus, Aurora, CO, USA; 'Department of Medicine, University of Minnesota, Minneapolis, MN, USA; 'Department of Internal Medicine, Wake Forest School of Medicine, Winston-Salem, NC, USA; 'Department of Medicine, Mayo Clinic, Jacksonville, FL, USA; 'Department of Laboratory Medicine and Pathology, University of Minnesota, Minneapolis, MN, USA; mPunzi Medical Center, Trinity Hypertension Research Institute, Carollton, TX, USA; ${ }^{n}$ Department of Medicine, Tufts Medical Center, Boston, MA, USA; ${ }^{\circ}$ Department of Medicine, University of Florida, Gainesville, FL, USA

\section{Keywords}

Urinary biomarkers - Uromodulin · $\beta 2$-microglobulin . a1-microglobulin . Chronic kidney disease

\begin{abstract}
Background: Kidney tubular atrophy on biopsy is a strong predictor of chronic kidney disease (CKD) progression, but tubular health is poorly quantified by traditional measures including estimated glomerular filtration rate (eGFR) and albuminuria. We hypothesized that urinary biomarkers of impaired tubule function would be associated with faster eGFR declines in persons with CKD. Methods: We measured baseline urine concentrations of uromodulin, $\beta 2$-microglobulin $(\beta 2 \mathrm{~m})$, and a1-microglobulin (a1m) among 2,428 partici-
\end{abstract}

pants of the Systolic Blood Pressure Intervention Trial with an eGFR $<60 \mathrm{~mL} / \mathrm{min} / 1.73 \mathrm{~m}^{2}$. We used linear mixed models to evaluate biomarker associations with annualized relative change in eGFR, stratified by randomization arm. Results: At baseline, the mean age was $73 \pm 9$ years and eGFR was $46 \pm$ $11 \mathrm{~mL} / \mathrm{min} / 1.73 \mathrm{~m}^{2}$. In the standard blood pressure treatment arm, each 2-fold higher urinary uromodulin was associated with slower \% annual eGFR decline $(0.34$ [95\% Cl: 0.08, $0.60])$, whereas higher urinary $\beta 2 \mathrm{~m}$ was associated with faster \% annual eGFR decline $(-0.10$ [95\% Cl: $-0.18,-0.02])$ in multivariable-adjusted models including baseline eGFR and albuminuria. Associations were weaker and did not reach

Co-first authors are Vasantha Jotwani and Pranav S. Garimella. Co-senior authors are Michael G. Shlipak and Joachim H. Ix. karger@karger.com www.karger.com/ajn

(C) 2020 S. Karger AG, Base

Karger'?
Vasantha Jotwani

Kidney Health Research Collaborative

4150 Clement Street, Bldg. 2

San Francisco, CA 94121 (USA)

vasantha.jotwani@ucsf.edu 
statistical significance in the intensive blood pressure treatment arm for either uromodulin $(0.11[-0.13,0.35], p$ value for interaction by treatment arm $=0.045)$ or $\beta 2 \mathrm{~m}(-0.01$ $[-0.08,0.08], p$ value for interaction $=0.001)$. Urinary a $1 \mathrm{~m}$ was not independently associated with eGFR decline in the standard $(0.01[-0.22,0.23])$ or intensive $(0.03[-0.20,0.25])$ arm. Conclusions: Among trial participants with hypertension and CKD, baseline measures of tubular function were associated with subsequent declines in kidney function, although these associations were diminished by intensive blood pressure control.

(c) 2020 S. Karger AG, Basel

\section{Introduction}

Kidney tubular atrophy and interstitial fibrosis on biopsy are strong predictors of chronic kidney disease (CKD) progression $[1,2]$. Unfortunately, tubular damage and function are inadequately captured by estimated glomerular filtration rate (eGFR) and albuminuria, which primarily indicate glomerular function and injury $[3,4]$. Several urinary proteins have emerged as promising biomarkers of kidney tubular health, with the goal of improving prognostication of CKD progression and its complications. Uromodulin or Tamm-Horsfall protein is synthesized by tubular epithelial cells in the thick ascending loop of Henle and the distal tubule [5]. Higher urine levels of uromodulin have been associated with lower risks of acute kidney injury (AKI), CKD, urinary tract infection, andmortality [6-9]. Bycontrast, $\beta 2$-microglobulin $(\beta 2 \mathrm{~m})$ and $\alpha 1$-microglobulin $(\alpha 1 \mathrm{~m})$ are low-molecularweight proteins that are filtered at the glomerulus and reabsorbed by proximal tubular epithelial cells $[10,11]$; therefore, higher urinary $\beta 2 \mathrm{~m}$ and $\alpha 1 \mathrm{~m}$ are early indicators of tubular dysfunction $[12,13]$. Higher urinary a $1 \mathrm{~m}$ levels have been associated with faster kidney function decline in HIV-infected and uninfected women, with graft failure and cardiovascular disease (CVD) risk in kidney transplant recipients, with higher mortality risk in elders, and with CVD risk in Systolic Blood Pressure Intervention Trial (SPRINT) participants with CKD [1417]. However, it is unclear whether these markers of tubular health are prognostic of subsequent declines in kidney function among persons with CKD.

The objective of this study was to evaluate the associations of urinary markers of kidney tubular function (uromodulin, $\beta 2 \mathrm{~m}$, and $\alpha 1 \mathrm{~m}$ ) with longitudinal changes in kidney function among SPRINT participants with CKD. We hypothesized that impairments in kidney tubular function, indicated by lower urinary uromodulin and higher urinary $\beta 2 \mathrm{~m}$ and $\alpha 1 \mathrm{~m}$ levels, would be associated with faster declines in kidney function during follow-up, independent of baseline eGFR and albuminuria.

\section{Materials and Methods}

\section{Study Design and Participants}

SPRINT was an open-label clinical trial that randomized persons with systolic blood pressure (SBP) $>130 \mathrm{~mm} \mathrm{Hg}$ and elevated risk for CVD events to an SBP target of $<120 \mathrm{~mm} \mathrm{Hg}$ ("intensive") versus $<140 \mathrm{~mm} \mathrm{Hg}$ ("standard”) [18]. Trial results were published previously $[19,20]$. Participants were recruited from 102 centers in the USA and Puerto Rico and were required to meet the following inclusion criteria: age $\geq 50$ years, SBP $130-180 \mathrm{~mm} \mathrm{Hg}$ depending on the number of antihypertensive medications, and increased risk for CVD events (defined by: prior clinical or subclinical CVD other than stroke, 10-year risk of CVD of $15 \%$ or greater based on the Framingham risk score [21], eGFR $20-59 \mathrm{~mL} / \mathrm{min} / 1.73 \mathrm{~m}^{2}$, or age $\geq 75$ years). Major exclusion criteria included diabetes mellitus, proteinuria $>1 \mathrm{~g} /$ day, polycystic kidney disease, prior stroke or transient ischemic attack, symptomatic heart failure, or a left ventricular ejection fraction $<35 \%$. A total of 9,361 participants were enrolled between November 2010 and March 2013. SPRINT randomly assigned participants in a 1:1 ratio to the intensive or standard blood pressure arm. The antihypertensive regimens were adjusted to maintain SBP according to the randomized treatment target. Participants attended visits monthly during the phase of antihypertensive medication titration and every 3 months thereafter; clinical data were obtained at serial visits. Venous blood and urine specimens were processed immediately, shipped overnight on dry ice, and stored at $-80^{\circ} \mathrm{C}$ at the SPRINT Central Laboratory at the University of Minnesota. All participants provided written informed consent, and Institutional Review Boards of all participating institutions approved the study. The present study was conducted in accordance with the Declaration of Helsinki and was also approved by the committees on human research at the University of California, San Francisco, and the Veteran Affairs San Francisco Health Care System and the Veterans Affairs San Diego Health Care System.

We measured urinary biomarkers at the baseline visit among the 2,514 SPRINT participants with eGFR $<60 \mathrm{~mL} / \mathrm{min} / 1.73 \mathrm{~m}^{2}$, defined by the CKD-EPI (Chronic Kidney Disease Epidemiology Collaboration) equation that uses both serum creatinine and cystatin C [22]. Among these participants, 86 were excluded due to unavailable urine specimens or invalid urinary biomarker measurements, resulting in a final analytic sample of 2,428 participants.

Exposures

Urinary uromodulin, $\beta 2$-microglobulin $(\beta 2 \mathrm{~m})$, and $\alpha 1$ microglobulin $(\alpha 1 \mathrm{~m})$ were measured at the Laboratory for Clinical Biochemistry Research at the University of Vermont. All urine specimens were in continuous storage at $-80^{\circ} \mathrm{C}$ until biomarker measurement without prior thaw. Laboratory personnel performing the biomarker assays were blinded to clinical information about the participants. Urine $\beta 2 \mathrm{~m}$ and uromodulin were performed by multiplex assays (Meso Scale Diagnostics, Rockville, MD, USA) with detectable ranges of $1.2-5,020$ and $0.6-2,510 \mathrm{ng} /$ $\mathrm{mL}$, respectively. Urine $\alpha 1 \mathrm{~m}$ was measured by a nephelometric as- 
Table 1. Baseline characteristics of SPRINT participants with CKD by the randomized treatment arm $(N=2,428)$

\begin{tabular}{|c|c|c|}
\hline & $\begin{array}{l}\text { Standard BP arm } \\
N=1,186\end{array}$ & $\begin{array}{l}\text { Intensive BP arm } \\
N=1,242\end{array}$ \\
\hline Age at randomization & $73(9)$ & $73(9)$ \\
\hline Female & $479(40)$ & $500(40)$ \\
\hline \multicolumn{3}{|l|}{ Race } \\
\hline Non-Hispanic white & $792(67)$ & $813(66)$ \\
\hline Non-Hispanic black & $299(25)$ & $322(26)$ \\
\hline Hispanic & $79(7)$ & $85(7)$ \\
\hline Other & $16(1)$ & $22(2)$ \\
\hline History of cardiovascular disease & $302(26)$ & $309(25)$ \\
\hline \multicolumn{3}{|l|}{ Smoking status } \\
\hline Never & $547(46)$ & $554(45)$ \\
\hline Former & $538(45)$ & $577(47)$ \\
\hline Current & $101(9)$ & $111(9)$ \\
\hline Systolic blood pressure, $\mathrm{mm} \mathrm{Hg}$ & $140(16)$ & $139(16)$ \\
\hline Diastolic blood pressure, $\mathrm{mm} \mathrm{Hg}$ & $74(12)$ & $75(12)$ \\
\hline \multicolumn{3}{|c|}{ Antihypertensive medications at baseline, $n$} \\
\hline 0 & $50(4)$ & $50(4)$ \\
\hline 1 & $267(23)$ & $277(22)$ \\
\hline 2 & $419(35)$ & $461(37)$ \\
\hline 3 & $344(29)$ & $349(28)$ \\
\hline$\geq 4$ & $106(9)$ & $105(9)$ \\
\hline Body mass index, $\mathrm{kg} / \mathrm{m}^{2}$ & $29.4(5.8)$ & $29.6(5.9)$ \\
\hline Total cholesterol, mg/dL & $183(40)$ & $184(41)$ \\
\hline High-density lipoprotein, mg/dL & $52(14)$ & $53(15)$ \\
\hline Triglycerides, mg/dL & $113[83,156]$ & $111[79,150]$ \\
\hline $\mathrm{eGFR}, \mathrm{mL} / \mathrm{min} / 1.73 \mathrm{~m}^{2}$ & $46(11)$ & $46(11)$ \\
\hline Urine albumin/creatinine ratio, $\mathrm{mg} / \mathrm{g}$ & $15[7,50]$ & $14[7,46]$ \\
\hline Urine uromodulin, $\mathrm{ng} / \mathrm{mL}$ & $6.5[4.4,9.9]$ & $6.6[4.2,10.2]$ \\
\hline Urine $\beta 2$-microglobulin, $\mathrm{ng} / \mathrm{mL}$ & $95[35,317]$ & $99[32,327]$ \\
\hline Urine a1-microglobulin, mg/L & $14[7,26]$ & $13[7,24]$ \\
\hline
\end{tabular}

Data are presented as median (interquartile range), mean \pm standard deviation, or $\mathrm{n}(\%)$. BP, blood pressure; $\mathrm{CKD}$, chronic kidney disease; eGFR, estimated glomerular filtration rate by the combined CKD-EPI equation for serum creatinine and cystatin C.

say (Siemens, Tarrytown, NY, USA) with a detectable range of $5-480 \mathrm{mg} / \mathrm{L}$. Interassay coefficients of variation for the urine measurements were $13-16 \%$ for uromodulin, $15-16 \%$ for $\beta 2 \mathrm{~m}$, and $3.5-8.8 \%$ for $a 1 \mathrm{~m}$. Biomarkers were measured in duplicate and averaged to increase precision. Urine creatinine was measured by an enzymatic procedure (Roche, Indianapolis, IN, USA) and urine albumin by a nephelometric method (Siemens, Tarrytown, NY, USA). Interassay coefficients of variation for urine creatinine and albumin measurements were $1.5-4.3 \%$ and $2.2-6.9 \%$, respectively. Samples with biomarker values below the limit of detection were assigned a value equivalent to the lower limit of detection divided by the square root of 2 [23].

\section{Outcomes}

Glomerular filtration was assessed every 3 months by serum creatinine, which was measured at the SPRINT Central Laboratory at the University of Minnesota. eGFR was calculated by the CKD-EPI equation for creatinine for follow-up assessments in
SPRINT. The outcomes of this study included (1) annualized relative eGFR change and (2) a prespecified kidney composite outcome of the SPRINT parent study protocol, defined as $\geq 50 \%$ eGFR decline (confirmed by repeat testing $\geq 90$ days) or end-stage kidney disease (ESKD) requiring dialysis or kidney transplantation [20].

\section{Statistical Analysis}

Spearman coefficients were used to evaluate correlations among the urinary biomarkers, urine albumin, urine creatinine, and eGFR. We used linear mixed models and Cox proportional hazards models to evaluate associations of each urinary biomarker with annualized eGFR change and the composite kidney outcome, respectively. Each urinary biomarker was evaluated as a continuous variable using the log base 2-transformation, providing estimates "per 2-fold higher value" of each marker. Participants were censored at death or last available study follow-up. Covariates for multivariable models were selected based on biological plausibility and included age, sex, race (non-Hispanic white, non-Hispanic black, and others), 


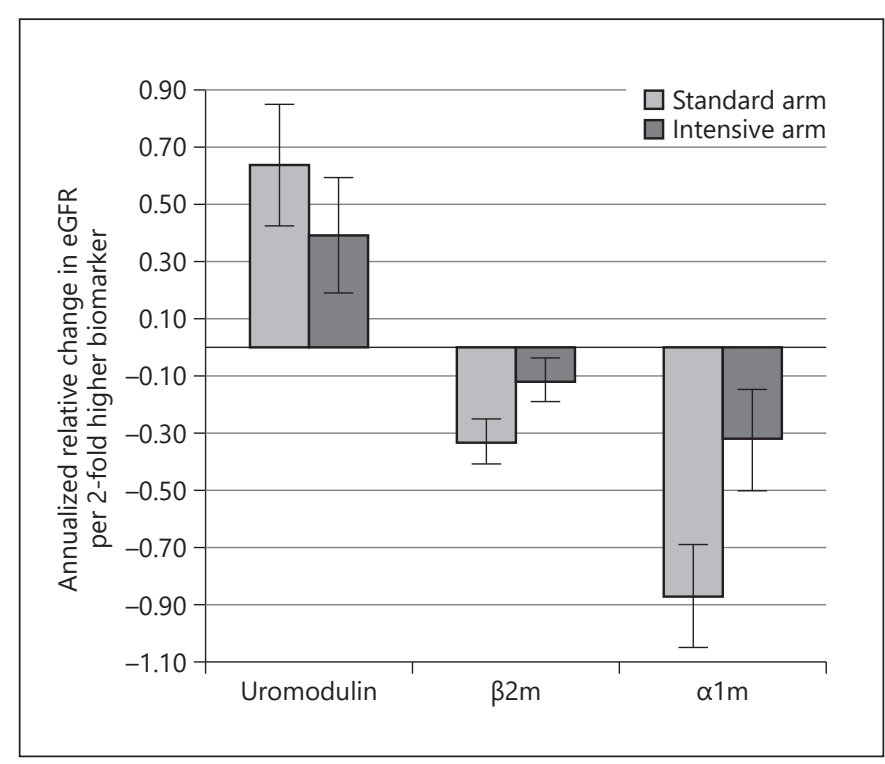

Fig. 1. Unadjusted associations of baseline urinary biomarker concentrations and annualized relative eGFR change among SPRINT participants with CKD, stratified by randomization arm. Estimates are displayed per 2 -fold higher value (95\% confidence interval) of baseline biomarker concentration.

urine creatinine, eGFR, urine albumin, smoking status (current, former, and never), history of CVD or heart failure, number of antihypertensive medications, systolic and diastolic blood pressures, body mass index, high-density lipoprotein, and total cholesterol. Baseline values were used for all covariates. Because urine creatinine is influenced by muscle mass and health status and may therefore influence the ratio of biomarkers to creatinine [24], our primary analyses evaluated biomarker concentrations that adjusted for urine creatinine concentration in the multivariable models. In secondary analyses, the biomarkers were indexed to urine creatinine as biomarker/creatinine ratios. Because blood pressure targets may alter urine levels of tubule function markers [25] and exert hemodynamic effects on eGFR, we stratified the analyses by randomization blood pressure arm. We also tested multiplicative interaction terms between each biomarker and treatment assignment for each outcome. All analyses were conducted using Stata (Stata Statistical Software, release 13; StataCorp LP, College Station, TX, USA) and SPSS (released 2016, IBM SPSS Statistics for Windows, Version 24.0; IBM Corp., Armonk, NY, USA).

\section{Results}

Baseline Characteristics of SPRINT CKD Participants

Among the 2,428 participants included in this study (Table 1), 1,186 participants were randomized to the standard systolic blood pressure treatment arm and 1,242 participants were randomized to the intensive systolic blood pressure treatment arm. The mean age was $73 \pm 9$ years, and $40 \%$ of participants were women. Non-Hispanic blacks comprised $26 \%$ of participants, and CVD or heart failure was prevalent among $25 \%$ of participants at baseline. The mean eGFR was $46 \pm 11 \mathrm{~mL} / \mathrm{min} / 1.73 \mathrm{~m}^{2}$, and the median urine albumin/creatinine ratio was $15 \mathrm{mg} / \mathrm{g}$ (interquartile range: 7, 47). Baseline demographic and clinical characteristics were similar between blood pressure treatment arms. The distributions of all 3 urinary biomarkers were right-skewed; median values (interquartile range) were $6.5 \mathrm{ng} / \mathrm{mL}(4.4,9.9)$ for uromodulin, $95 \mathrm{ng} /$ $\mathrm{mL}(35,317)$ for $\beta 2 \mathrm{~m}$, and $14 \mathrm{mg} / \mathrm{L}(7,26)$ for $\alpha 1 \mathrm{~m}$.

\section{Correlations of Urinary Biomarkers and eGFR}

Urinary uromodulin was positively correlated with eGFR (see online suppl. Table 1; for all online suppl. material, see www.karger.com/doi/10.1159/000509978; $r=$ 0.35 ) but showed little correlation with urinary $\beta 2 \mathrm{~m}$, $\alpha 1 \mathrm{~m}$, or albumin $(r<0.01$ to 0.10$)$. Urinary $\beta 2 \mathrm{~m}$ and $\alpha 1 \mathrm{~m}$ were inversely correlated with eGFR $(r=-0.13$ and -0.22 , respectively), positively correlated with urine albumin $(r=0.27$ and 0.52 , respectively), and positively correlated with each other $(r=0.50)$.

\section{Associations of Kidney Tubule Function with Longitudinal Changes in eGFR}

Participants underwent a mean of $8.0 \pm 2.3$ eGFR assessments over a median follow-up of 3.3 years (25th75th percentile, 2.8-3.8). Compared to the standard arm, the intensive group had a slightly faster rate of eGFR decline by $0.15 \mathrm{~mL} / \mathrm{min} / 1.73 \mathrm{~m}^{2}$ per year after the initial 6 months $[19,20]$. In unadjusted models, lower urine uromodulin levels and higher urine $\beta 2 \mathrm{~m}$ and $\alpha 1 \mathrm{~m}$ levels were associated with faster eGFR declines in both standard and intensive treatment arms (Fig. 1). After multivariable adjustment for baseline eGFR and traditional CKD risk factors (Table 2), lower uromodulin and higher $\beta 2 \mathrm{~m}$ and $\alpha 1 \mathrm{~m}$ were independently associated with faster eGFR declines in the standard treatment arm. With additional adjustment for albuminuria, lower uromodulin and higher $\beta 2 \mathrm{~m}$ remained associated with faster eGFR decline, but the association of urine $\alpha 1 \mathrm{~m}$ with eGFR change was largely attenuated and no longer statistically significant.

In the intensive treatment arm, lower uromodulin and higher $\beta 2 \mathrm{~m}$ and $\alpha 1 \mathrm{~m}$ levels were each associated with faster eGFR declines in demographic-adjusted models. After multivariable adjustment for baseline eGFR and traditional CKD risk factors, higher urine $\alpha 1 \mathrm{~m}$ remained associated with faster eGFR decline, but the association was attenuated substantially after further adjustment for albuminuria. Associations of uromodulin and $\beta 2 \mathrm{~m}$ with 
Table 2. Associations of baseline tubule function biomarkers with annualized eGFR change among SPRINT participants with CKD $(N=2,428)$

\begin{tabular}{|c|c|c|c|}
\hline & \multicolumn{2}{|c|}{$\%$ annual eGFR change $(95 \% \mathrm{CI})$} & \multirow{2}{*}{$\begin{array}{l}p \text { value for } \\
\text { interaction }\end{array}$} \\
\hline & $\begin{array}{l}\text { Standard BP arm } \\
N=1,186\end{array}$ & $\begin{array}{l}\text { Intensive BP arm } \\
N=1,242\end{array}$ & \\
\hline \multicolumn{4}{|c|}{ Urine uromodulin (per 2-fold higher) } \\
\hline Model $1^{\mathrm{a}}$ & $0.60(0.35,0.85)$ & $0.36(0.13,0.58)$ & 0.090 \\
\hline Model $2^{\mathrm{b}}$ & $0.34(0.08,0.60)$ & $0.08(-0.16,0.32)$ & 0.019 \\
\hline Model $3^{c}$ & $0.34(0.08,0.60)$ & $0.11(-0.13,0.35)$ & 0.045 \\
\hline \multicolumn{4}{|c|}{ Urine $\beta 2 \mathrm{~m}$ (per 2 -fold higher) } \\
\hline Model $1^{\mathrm{a}}$ & $-0.31(-0.39,-0.23)$ & $-0.12(-0.20,-0.04)$ & $<0.001$ \\
\hline Model $2^{\mathrm{b}}$ & $-0.20(-0.28,-0.12)$ & $-0.07(-0.15,0.01)$ & 0.002 \\
\hline Model $3^{c}$ & $-0.10(-0.18,-0.02)$ & $-0.01(-0.08,0.08)$ & 0.001 \\
\hline \multicolumn{4}{|c|}{ Urine a $1 \mathrm{~m}$ (per 2-fold higher) } \\
\hline Model $1^{\mathrm{a}}$ & $-1.07(-1.26,-0.87)$ & $-0.53(-0.72,-0.34)$ & $<0.001$ \\
\hline Model $2^{\mathrm{b}}$ & $-0.57(-0.78,-0.36)$ & $-0.28(-0.49,-0.07)$ & $<0.001$ \\
\hline Model $3^{c}$ & $0.01(-0.22,0.23)$ & $0.03(-0.20,0.25)$ & 0.71 \\
\hline
\end{tabular}

alm, a1-microglobulin; $\beta 2 \mathrm{~m}, \beta 2$-microglobulin; BP, blood pressure; CKD, chronic kidney disease; eGFR, estimated glomerular filtration rate. ${ }^{a}$ Adjusted for age, sex, race, and urine creatinine. ${ }^{\mathrm{b}}$ Adjusted for model 1 covariates and baseline eGFR, smoking status, history of cardiovascular disease, baseline number of antihypertensive medications, systolic blood pressure, diastolic blood pressure, body mass index, high-density lipoprotein, and total cholesterol. ${ }^{c}$ Adjusted for model 2 covariates and urine albumin.

eGFR change were also attenuated by multivariable adjustment, and neither had independent associations in fully adjusted models. Statistically significant interactions by randomization arm were observed for the associations of uromodulin $(p=0.045)$ and $\beta 2 \mathrm{~m}(p=0.001)$ with eGFR change; larger effect estimates were present in the standard arm as compared to the intensive arm.

Findings were similar when urine biomarkers were standardized to urine creatinine (online suppl. Table 2). In fully adjusted models that included baseline eGFR, albuminuria, and traditional CKD risk factors, lower uromodulin/cr ( $0.26 \%$ change per year; $95 \%$ CI: $0.01,0.51)$ and higher $\beta 2 \mathrm{~m} / \mathrm{cr}(-0.08$; 95\% CI: $-0.16,-0.01)$ were each independently associated with faster eGFR declines in the standard treatment arm, but not in the intensive arm. Urine $\alpha 1 \mathrm{~m} / \mathrm{cr}$ was not associated with eGFR change in either treatment arm in fully adjusted models.

\section{Associations of Kidney Tubule Function with the Composite Kidney Outcome}

Over a median follow-up of 3.8 years, there were 87 composite kidney events (Table 3 ). Among these events, 30 occurred in the standard arm (26 cases of $\geq 50 \%$ eGFR decline and 4 ESKD events) and 57 occurred in the intensive arm (54 cases of $\geq 50 \%$ eGFR decline and 3 ESKD events). In multivariable-adjusted models that included demo- graphics, CKD risk factors, baseline eGFR, and albuminuria, each 2 -fold higher level of urine uromodulin was associated with $31 \%$ lower risk of the composite kidney outcome in the standard arm and $17 \%$ lower risk in the intensive arm ( $p$ value for interaction $=0.032$ ). Urine $\beta 2 \mathrm{~m}$ levels were not associated with the composite kidney outcome in either treatment arm in multivariable-adjusted models. In the standard treatment arm, each 2 -fold higher urine $\alpha 1 \mathrm{~m}$ was associated with a $48 \%$ higher risk of the composite kidney outcome in models that adjusted for baseline eGFR and traditional CKD risk factors. However, after additional adjustment for albuminuria, the association was moderately attenuated and was no longer statistically significant. In the intensive treatment arm, urine $\alpha 1 \mathrm{~m}$ appeared to have no association with the composite kidney outcome in multivariable models ( $p$ value for interaction by treatment arm $=0.008$ ).

Findings were similar when the biomarkers were standardized to urine creatinine, with none of the markers showing independent associations with the composite kidney outcome in either treatment arm in fully adjusted models (online suppl. Table 3). When participants were stratified by age $<75$ or $\geq 75$ years, there were no significant interactions for the associations of the urine biomarkers with eGFR change or the composite kidney outcome ( $p$ value $\geq 0.05$ for all biomarkers). 
Table 3. Associations of baseline tubule function biomarkers with $50 \%$ eGFR decline or ESKD requiring dialysis or transplant among SPRINT participants with CKD $(N=2,428)$

\begin{tabular}{|c|c|c|c|}
\hline & \multicolumn{2}{|c|}{ Hazard ratio $(95 \% \mathrm{CI})$} & \multirow{2}{*}{$\begin{array}{l}p \text { value for } \\
\text { interaction }\end{array}$} \\
\hline & $\begin{array}{l}\text { Standard BP arm } \\
N=30 \text { events }\end{array}$ & $\begin{array}{l}\text { Intensive BP arm } \\
N=57 \text { events }\end{array}$ & \\
\hline \multicolumn{4}{|c|}{ Urine uromodulin (per 2-fold higher) } \\
\hline Model $1^{\mathrm{a}}$ & $0.56(0.39,1.79)$ & $0.74(0.59,0.94)$ & 0.039 \\
\hline Model $2^{\mathrm{b}}$ & $0.79(0.55,1.15)$ & $0.88(0.69,1.12)$ & 0.035 \\
\hline Model $3^{c}$ & $0.69(0.47,1.02)$ & $0.83(0.64,1.09)$ & 0.032 \\
\hline \multicolumn{4}{|c|}{ Urine $\beta 2 \mathrm{~m}$ (per 2-fold higher) } \\
\hline Model $1^{\mathrm{a}}$ & $1.23(1.03,1.45)$ & $1.04(0.94,1.16)$ & 0.085 \\
\hline Model $2^{\mathrm{b}}$ & $1.04(0.88,1.22)$ & $1.02(0.92,1.12)$ & 0.220 \\
\hline Model $3^{c}$ & $1.00(0.85,1.19)$ & $0.98(0.89,1.08)$ & 0.213 \\
\hline \multicolumn{4}{|c|}{ Urine a $1 \mathrm{~m}$ (per 2-fold higher) } \\
\hline Model $1^{\mathrm{a}}$ & $2.40(1.73,3.33)$ & $1.38(1.09,1.74)$ & 0.010 \\
\hline Model $2^{\mathrm{b}}$ & $1.48(1.07,2.03)$ & $1.14(0.87,1.50)$ & 0.011 \\
\hline Model $3^{c}$ & $1.29(0.91,1.82)$ & $0.94(0.71,1.26)$ & 0.008 \\
\hline
\end{tabular}

a1m, a1-microglobulin; $\beta 2 \mathrm{~m}, \beta 2$-microglobulin; BP, blood pressure; CKD, chronic kidney disease; eGFR, estimated glomerular filtration rate; ESKD, end-stage kidney disease; HR, hazard ratio. a Adjusted for age, sex, race, and urine creatinine. ${ }^{b}$ Adjusted for model 1 covariates and baseline eGFR, smoking status, history of cardiovascular disease, baseline number of antihypertensive medications, systolic blood pressure, diastolic blood pressure, body mass index, high-density lipoprotein, and total cholesterol. ${ }^{\mathrm{c}}$ Adjusted for model 2 covariates and urine albumin.

\section{Discussion}

Kidney tubules are essential for solute transport, acidbase homeostasis, regulation of volume status, and elimination of toxins [26]. Dysfunction of the kidney tubules is an early feature of kidney injury from ischemic, toxic, or inflammatory insults, and tubular damage on biopsy is a strong predictor of subsequent CKD progression [2630]. However, current clinical tests of kidney health primarily assess glomerular filtration (eGFR) and injury (albuminuria). In this ancillary study of SPRINT participants with CKD at baseline, we hypothesized that 3 urinary markers of kidney tubular function, uromodulin, $\beta 2 \mathrm{~m}$, and $\alpha 1 \mathrm{~m}$, would be associated with loss of eGFR and risk of CKD progression, independent of baseline eGFR and albuminuria. We found that lower uromodulin and higher $\beta 2 \mathrm{~m}$ levels were each independently associated with faster eGFR decline in the standard blood pressure treatment arm. Directions of these associations are both consistent with the hypothesis that worse tubule health at baseline is a risk factor for faster declines in eGFR. By contrast, associations of uromodulin and $\beta 2 \mathrm{~m}$ with subsequent changes in kidney function were markedly weaker in the intensive systolic blood pressure treatment arm with significant biomarker by intervention arm interac- tions. Lower uromodulin also appeared to be associated with higher risk of the composite kidney outcome, but the associations did not reach statistical significance after multivariable adjustment including baseline eGFR and albuminuria. There were no significant adjusted associations of urine a $1 \mathrm{~m}$ with eGFR change or with the composite kidney outcome in fully adjusted models.

The beneficial association of higher urine uromodulin with lower risk of subsequent kidney dysfunction is supported by several epidemiological studies conducted in ambulatory and hospitalized individuals. In a case-cohort study within the Cardiovascular Health Study, each standard deviation higher urinary uromodulin was associated with $23 \%$ lower odds ( $95 \%$ CI: $38,4 \%$ ) of $\geq 30 \%$ eGFR decline, over approximately 10 years of follow-up [6]. Another case-cohort study conducted within the Health, Aging and Body Composition study found that persons in the highest quartile of urinary uromodulin had a 53\% lower risk (95\% CI: 73\%, 18\%) of incident CKD and a 51\% lower risk of rapidly declining kidney function (95\% CI: $76 \%, 1 \%)$ compared with the lowest uromodulin quartile [9]. Higher urinary uromodulin levels have also been associated with lower risks of AKI and urinary tract infection in prior studies $[7,8]$; within the SPRINT trial, we recently reported that higher urine uromodulin levels 
were associated with lower risk of future AKI events [31]. Furthermore, in a study of 527 individuals with type 1 diabetes mellitus, serum uromodulin levels were independently associated with incident albuminuria and incident eGFR $<60 \mathrm{~mL} / \mathrm{min} / 1.73 \mathrm{~m}^{2}$ [32]. UMOD gene knockout mice develop more extensive ischemic AKI, suggesting that this protein may be mechanistically linked with risk of subsequent loss of kidney function $[33,34]$. Our current study builds upon the growing body of evidence indicating a protective role of uromodulin in kidney health. Further research is needed to determine whether urine levels of uromodulin are markers of kidney tubular health overall, or rather are causally protective to the kidney tubules.

Few prior studies have examined associations of lowmolecular-weight proteins such as urine $\beta 2 \mathrm{~m}$ and $\alpha 1 \mathrm{~m}$ with the risk of CKD progression. In the Women's Interagency HIV Study (WIHS), women with HIV in the highest tertile of urinary a $1 \mathrm{~m}$ had a 2 -fold higher risk of incident CKD and 3-fold higher risk of rapid eGFR decline compared with the lowest tertile, after adjustment for CKD risk factors, baseline eGFR, and albuminuria [14]. Among women without HIV in WIHS, those in the highest tertile also had a 2 -fold higher risk of rapid eGFR decline during follow-up, compared with the lowest urinary $\alpha 1 \mathrm{~m}$ tertile. Furthermore, in a study of ambulatory kidney transplant recipients, higher urinary a $1 \mathrm{~m}$ levels were associated with an approximately 2 -fold higher risk of allograft failure independent of eGFR and albuminuria [15]. In the present study of SPRINT participants with $\mathrm{CKD}$, we observed that higher urinary $\beta 2 \mathrm{~m}$ and alm levels were each associated with faster eGFR declines in multivariable models that included traditional CKD risk factors and baseline eGFR. However, adjustment for albuminuria attenuated these associations moderately, and only urine $\beta 2 \mathrm{~m}$ remained significantly associated with eGFR change in the fully adjusted model. As low-molecular-weight proteins, $\beta 2 \mathrm{~m}$ and $\alpha 1 \mathrm{~m}$ are freely filtered at the glomerulus and undergo active uptake by the megalin transport system within the proximal tubule, with elevated urine levels indicating impaired tubular reabsorptive capacity [11]. Because albumin is partially filtered and reabsorbed by the same cubilin-megalin system $[35,36]$, we hypothesize that adjustment for albumin may have weakened the observed associations of urine $\beta 2 \mathrm{~m}$ and $\alpha 1 \mathrm{~m}$ with subsequent declines in kidney function. This hypothesis is supported by the strong correlation of urine albumin with $\alpha 1 \mathrm{~m}$ and raises the possibility that the associations of albuminuria with eGFR decline may be due, in part, to proximal tubule dysfunction. Together with the protective associations of urinary uromodulin with eGFR change, and in the context of prior literature, we believe that our findings confirm the importance of impaired kidney tubule function as a risk factor for subsequent kidney disease progression.

Associations of urine uromodulin and $\beta 2 \mathrm{~m}$ appeared stronger in the standard blood pressure arm, compared with the intensive arm. The observed interactions by randomization arm may be explained by effects of blood pressure targets on the exposures or the outcomes of this study. In a subset of SPRINT CKD participants $(N=978)$, we previously found that $\alpha 1 \mathrm{~m}$ and $\beta 2 \mathrm{~m}$ were lower at year 1 in the intensive arm, compared with the standard arm [25]. Additionally, over the first 6 months of the SPRINT trial, larger declines in eGFR were observed in the intensive arm, as compared with the standard arm [20,37]. These observations suggest that blood pressure reduction may exert effects on urinary markers of tubule function and on eGFR. Because urine specimens were not available at the 6-month visit, our study could not determine associations of tubular health with longitudinal kidney outcomes after the initial phase of blood pressure reduction. Our data suggest that tubular function is an independent predictor of subsequent kidney health among participants randomized to standard blood pressure targets. Further studies will be needed to understand the interrelationships of intensive blood pressure control, kidney tubular health, and eGFR.

There are several limitations of this study. First, our results may not be generalizable to younger persons, individuals with diabetes mellitus, or persons with earlier stages of kidney damage and to those with severe proteinuria. Second, although we did not observe statistically significant associations for the composite kidney outcome, the study was underpowered given the low number of events. Third, the nephelometric method lacks sensitivity for detection of $\alpha 1 \mathrm{~m}$ at lower concentrations and might have contributed to the null associations. Fourth, we were unable to examine effect modification by specific antihypertensive medication classes, which may have differing effects on proteinuria and eGFR. Finally, although we adjusted for potential confounders in our multivariable models, we cannot exclude the possibility of residual confounding.

In summary, among hypertensive individuals with $\mathrm{CKD}$, lower urinary uromodulin and higher urinary $\beta 2 \mathrm{~m}$ were each associated with faster eGFR declines among participants randomized to a standard blood pressure target. These associations were independent of baseline eGFR and 
albuminuria levels, suggesting that impaired kidney tubular function may be an important risk factor for eGFR decline. Further studies are needed to validate these findings and to investigate the clinical utility of these urinary biomarkers for monitoring of kidney tubular health.

\section{Statement of Ethics}

All participants provided written informed consent, and Institutional Review Boards of all participating institutions approved the study. The present study was conducted in accordance with the Declaration of Helsinki and was also approved by the committees on human research at the University of California, San Francisco and the Veteran Affairs San Francisco Health Care System and the Veterans Affairs San Diego Health Care System.

\section{Conflicts of Interest Statement}

M.G.S. has worked as a consultant for the University of Washington and has equity in TAI Diagnostics and Cricket Health, Inc. J.H.I. holds an investigator initiated research grant from Baxter International Inc. B.I.F. is a consultant for AstraZeneca and Renalytix AI Pharmaceuticals. P.S.G. has received speaker fees from Otsuka. The results presented in this paper have not been published previously in whole or part, except in abstract form.

\section{Author Contributions}

Research idea and study design: M.G.S. and J.H.I.; data acquisition: J.B., A.K.C., M.C., P.E.D., B.I.F., W.E.H., A.A.K., and H.P.; data analysis/interpretation: V.J., R.K., P.S.G., R.M., M.J.S., M.S.S., J.B., A.K.C., M.C., P.E.D., B.I.F., W.E.H., A.A.K., H.P., J.H.I., and M.G.S.; statistical analysis: R.K.; supervision or mentorship: J.H.I. and M.G.S.; manuscript preparation: V.J., P.S.G., J.H.I., and M.G.S. Each author contributed important intellectual content during manuscript drafting or revision, accepts personal accountability for the author's own contributions, and agrees to ensure that questions pertaining to the accuracy or integrity of any portion of the work are appropriately investigated and resolved.

\section{Acknowledgements and Funding Sources}

ThisancillarystudywassupportedbytheNIDDK(K23DK109868 for V.J., K23DK114556 for P.S.G., R01DK098234 for M.G.S./J.H.I., and K24DK110427 for J.H.I.) and the American Heart Association (14EIA18560026 for J.H.I.). The authors thank the participants and staff members of the Systolic Blood Pressure Intervention Trial, which was funded with Federal funds from the National Institutes of Health (NIH), including the National Heart, Lung, and Blood Institute (NHLBI), the National Institute of Diabetes and Digestive and Kidney Diseases (NIDDK), the National Institute on Aging (NIA), and the National Institute of Neurological Disorders and Stroke (NINDS), under Contract Numbers HHSN268200900040C, HHSN268200900046C, HHSN268200900047C, HHSN268200900048C, and HHSN268200900049C and Inter-Agency Agreement Number A-HL-13-002-001. It was also supported in part with resources and use of facilities through the Department of Veterans Affairs. The SPRINT investigators acknowledge the contribution of study medications (azilsartan and azilsartan combined with chlorthalidone) from Takeda Pharmaceuticals International, Inc. All components of the SPRINT study protocol were designed and implemented by the investigators. The investigative team collected, analyzed, and interpreted the data. All aspects of manuscript writing and revision were carried out by the co-authors. The content is solely the responsibility of the authors and does not necessarily represent the official views of the NIH, the U.S. Department of Veterans Affairs, or the United States Government. For a full list of contributors to SPRINT, please see the supplementary acknowledgement list: https://www.sprinttrial.org/public/dspScience.cfm. We also acknowledge the support from the following CTSAs funded by NCATS: CWRU: UL1TR000439; OSU: UL1RR025755; U Penn: UL1RR024134 and UL1TR000003; Boston: UL1RR025771; Stanford: UL1TR000093; Tufts: UL1RR025752, UL1TR000073, and UL1TR001064; University of Illinois: UL1TR000050; University of Pittsburgh: UL1TR000005; UT Southwestern: 9U54TR000017-06; University of Utah: UL1TR000105-05; Vanderbilt University: UL1 TR000445; George Washington University: UL1TR000075; University of CA, Davis: UL1 TR000002; University of Florida: UL1 TR000064; University of Michigan: UL1TR000433; Tulane University: P30GM103337 COBRE Award NIGMS; Wake Forest University: UL1TR001420.

\section{References}

1 Howie AJ, Ferreira MA, Adu D. Prognostic value of simple measurement of chronic damage in renal biopsy specimens. Nephrol Dial Transplant. 2001;16(6):1163-9.

2 Nath KA. Tubulointerstitial changes as a major determinant in the progression of renal damage. Am J Kidney Dis. 1992;20(1): $1-17$.

3 Kassirer JP. Clinical evaluation of kidney function: glomerular function. $N$ Engl J Med. 1971;285(7):385-9.

4 Rule AD, Amer H, Cornell LD, Taler SJ, Cosio FG, Kremers WK, et al. The association between age and nephrosclerosis on renal biop- sy among healthy adults. Ann Intern Med. 2010;152(9):561-7.

5 Garimella PS, Sarnak MJ. Uromodulin in kidney health and disease. Curr Opin Nephrol Hypertens. 2017;26(2):136-42.

6 Garimella PS, Biggs ML, Katz R, Ix JH, Bennett MR, Devarajan P, et al. Urinary uromodulin, kidney function, and cardiovascular disease in elderly adults. Kidney Int. 2015;88(5):1126-34.

7 Garimella PS, Bartz TM, Ix JH, Chonchol M, Shlipak MG, Devarajan P, et al. Urinary uromodulin and risk of urinary tract infections: the cardiovascular health study. Am J Kidney Dis. 2016;69(6):744.
8 Garimella PS, Jaber BL, Tighiouart H, Liangos O, Bennett MR, Devarajan P, et al. Association of preoperative urinary uromodulin with AKI after cardiac surgery. Clin J Am Soc Nephrol. 2017;12(1):10-8.

9 Garimella PS, Katz R, Ix JH, Fried LF, Kritchevsky SB, Devarajan P, et al. Association of urinary uromodulin with kidney function decline and mortality: the health $\mathrm{ABC}$ study. Clin Nephrol. 2017;87(6):278-86.

10 Akerström B, Lögdberg L, Berggård T, Osmark P, Lindqvist A. Alpha(1)-microglobulin: a yellow-brown lipocalin. Biochim Biophys Acta. 2000;1482(1-2):172-84. 
11 Leheste JR, Rolinski B, Vorum H, Hilpert J, Nykjaer A, Jacobsen C, et al. Megalin knockout mice as an animal model of low molecular weight proteinuria. Am J Pathol. 1999;155(4): 1361-70.

12 Weber MH, Verwiebe R. Alpha 1-microglobulin (protein $\mathrm{HC}$ ): features of a promising indicator of proximal tubular dysfunction. Eur J Clin Chem Clin Biochem. 1992;30(10):68391.

13 Christensen EI, Nielsen S. Structural and functional features of protein handling in the kidney proximal tubule. Semin Nephrol. 1991;11(4):414-39.

14 Jotwani V, Scherzer R, Abraham A, Estrella MM, Bennett M, Cohen MH, et al. Association of urine a1-microglobulin with kidney function decline and mortality in HIV-infected women. Clin J Am Soc Nephrol. 2015; 10(1):63-73.

15 Ix JH, Katz R, Bansal N, Foster M, Weiner DE, Tracy R, et al. Urine fibrosis markers and risk of allograft failure in kidney transplant recipients: a case-cohort ancillary study of the FAVORIT trial. Am J Kidney Dis. 2017;69(3): 410-9.

16 Jotwani V, Katz R, Ix JH, Gutiérrez OM, Bennett M, Parikh CR, et al. Urinary biomarkers of kidney tubular damage and risk of cardiovascular disease and mortality in elders. Am J Kidney Dis. 2018;72(2):205-13.

17 Garimella PS, Lee AK, Ambrosius WT, Bhatt U, Cheung AK, Chonchol M, et al. Markers of kidney tubule function and risk of cardiovascular disease events and mortality in the SPRINT trial. Eur Heart J. 2019;40(42):3486-93.

18 Ambrosius WT, Sink KM, Foy CG, Berlowitz DR, Cheung AK, Cushman WC, et al. The design and rationale of a multicenter clinical trial comparing two strategies for control of systolic blood pressure: the Systolic Blood Pressure Intervention Trial (SPRINT). Clin Trials. 2014;11(5):532-46.

19 Group SR, Wright JT Jr, Williamson JD, Whelton PK, Snyder JK, Sink KM, et al. A randomized trial of intensive versus standard blood-pressure control. N Engl J Med. 2015; 373(22):2103-16.
20 Cheung AK, Rahman M, Reboussin DM, Craven TE, Greene T, Kimmel PL, et al. Effects of intensive $\mathrm{BP}$ control in CKD. J Am Soc Nephrol. 2017;28(9):2812-23.

21 D’Agostino RB Sr, Vasan RS, Pencina MJ, Wolf PA, Cobain M, Massaro JM, et al. General cardiovascular risk profile for use in primary care: the Framingham Heart Study. Circulation. 2008;117(6):743-53.

22 Levey AS, Stevens LA, Schmid CH, Zhang YL, Castro AF, Feldman HI, et al. A new equation to estimate glomerular filtration rate. Ann Intern Med. 2009;150(9):604-12.

23 Hornung RW, Reed LD. Estimation of average concentration in the presence of nondetectable values. Appl Occup Environ Hyg. 1990;5(1):46-51.

$24 \mathrm{Ix} \mathrm{JH}$, de Boer IH, Wassel CL, Criqui MH, Shlipak MG, Whooley MA. Urinary creatinine excretion rate and mortality in persons with coronary artery disease: the Heart and Soul Study. Circulation. 2010;121(11):1295303.

25 Malhotra R, Craven T, Ambrosius WT, Killeen AA, Haley WE, Cheung AK, et al. Effects of intensive blood pressure lowering on kidney tubule injury in CKD: a longitudinal subgroup analysis in SPRINT. Am J Kidney Dis. 2019;73(1):21-30.

26 Brenner BM, Rector FC. Brenner \& Rector's the kidney. 8th ed. Philadelphia: Saunders Elsevier; 2008.

27 Hsu CY, Chertow GM, McCulloch CE, Fan D, Ordoñez JD, Go AS. Nonrecovery of kidney function and death after acute on chronic renal failure. Clin J Am Soc Nephrol. 2009;4(5): 891-8.

28 Lo LJ, Go AS, Chertow GM, McCulloch CE, Fan D, Ordoñez JD, et al. Dialysis-requiring acute renal failure increases the risk of progressive chronic kidney disease. Kidney Int. 2009;76(8):893-9.

29 Ishani A, Nelson D, Clothier B, Schult T, Nugent $\mathrm{S}$, Greer N, et al. The magnitude of acute serum creatinine increase after cardiac surgery and the risk of chronic kidney disease, progression of kidney disease, and death. Arch Intern Med. 2011;171(3):226-33.
30 Coca SG, Yusuf B, Shlipak MG, Garg AX, Parikh CR. Long-term risk of mortality and other adverse outcomes after acute kidney injury: a systematic review and meta-analysis. Am J Kidney Dis. 2009;53(6):961-73.

31 Bullen AL, Katz R, Lee AK, Anderson CAM, Cheung AK, Garimella PS, et al. The SPRINT trial suggests that markers of tubule cell function in the urine associate with risk of subsequent acute kidney injury while injury markers elevate after the injury. Kidney Int. 2019; 96(2):470-9.

32 Bjornstad P, Wiromrat P, Johnson RJ, Sippl R, Cherney DZI, Wong R, et al. Serum uromodulin predicts less coronary artery calcification and diabetic kidney disease over 12 years in adults with type 1 diabetes: the CACTI study. Diabetes Care. 2019;42(2):297-302.

33 El-Achkar TM, Wu XR, Rauchman M, McCracken R, Kiefer S, Dagher PC. TammHorsfall protein protects the kidney from ischemic injury by decreasing inflammation and altering TLR4 expression. Am J Physiol Renal Physiol. 2008;295(2):F534-544.

34 El-Achkar TM, McCracken R, Rauchman M, Heitmeier MR, Al-Aly Z, Dagher PC, et al. Tamm-Horsfall protein-deficient thick ascending limbs promote injury to neighboring S3 segments in an MIP-2-dependent mechanism. Am J Physiol Renal Physiol. 2011; 300(4):F999-1007.

35 Amsellem S, Gburek J, Hamard G, Nielsen R, Willnow TE, Devuyst O, et al. Cubilin is essential for albumin reabsorption in the renal proximal tubule. J Am Soc Nephrol. 2010; 21(11):1859-67.

36 Nielsen R, Christensen EI, Birn H. Megalin and cubilin in proximal tubule protein reabsorption: from experimental models to human disease. Kidney Int. 2016;89(1):58-67.

37 Beddhu S, Shen J, Cheung AK, Kimmel PL, Chertow GM, Wei G, et al. Implications of early decline in eGFR due to intensive BP control for cardiovascular outcomes in SPRINT. J Am Soc Nephrol. 2019;30(8):1523-33. 